\title{
CHEMISTRY IN THE LMC AND SMC
}

\author{
L.E.B. JOHANSSON \\ Onsala Space Observatory \\ S-439 92 Onsala, Sweden
}

\begin{abstract}
Molecular abundances derived in a sample of CO complexes in the Magellanic Clouds are discussed. With a possible exception of $\mathrm{HCO}^{+}$, the chemical compositions observed in this sample show a striking uniformity. The data indicate that the molecular concentrations are down by a factor of 5 in the SMC, relative those observed in a cloud associated with the H II region N159 in the LMC. A similar difference seems to exist within the LMC, between the 30 Doradus region and the N159 area. An estimate of the electron abundance in the $\mathrm{N} 159$ cloud is presented, based on a recent detection of $\mathrm{DCO}^{+}$.
\end{abstract}

\section{Introduction}

The Magellanic Clouds provide unique opportunities for studying the effects of elemental abundance changes on the interstellar medium. The lower abundances of heavy elements compared with the Galaxy (Dufour 1984) are also reflected in significantly lower dust-to-gas ratios (Koornneef 1984). These properties are expected to have impacts on the structure of the interstellar gas. The weak $\mathrm{CO}(1-0)$ emission in the Magellanic Clouds (see e.g. Rubio et al. 1993, Israel et al. 1993) and the high global [C II]/CO emission ratio observed in the LMC (Mochizuki et al. 1994) probably are manifestations of such structural changes. The observed properties suggest that PDRs occupy a larger fraction of the ISM in the Magellanic Clouds than in the Galaxy, a result of the reduced dust abundances and, accordingly, a deeper penetration of the UV radiation into the ISM (cf. Lequeux et al. 1994, Mochizuki et al. 1994, Poglitsch et al. 1995).

A direct consequence of lower elemental abundances should be lower molecular concentrations in general, but also changes in the chemical composition of the gas related to the structural changes - a higher degree of 
"PDR-chemistry" at the expense of "core-chemistry" (i.e. a chemistry not influenced by the incident UV field).

\section{Observed regions}

The molecular line data presented here were observed towards $\mathrm{CO}$ complexes selected from two areas in the LMC (see Fig.1 in Booth 1993): one area including the giant $\mathrm{H}$ II region 30 Doradus and one, somewhat to the south, which contains the H II regions N158C, N160 and N159. In the SMC, the data come from observations of the $\mathrm{CO}$ complex associated with the H II region N27 and the far-infrared source LIRS 49 (see Rubio et al. 1993).

The 30 Doradus nebula and its southern environments contain many objects which manifest past and on-going star formation, e.g. supernova remnants, bright stellar clusters of massive young stars, protostellar objects, and $\mathrm{OH}-$ and $\mathrm{H}_{2} \mathrm{O}$-masers. However, there seems to be a gradient in the north-south direction with respect to the ages of stars and $\mathrm{H}$ II regions, with the 30 Doradus nebula being the most evolved one while the surroundings of N159 define the border of current massive star formation (cf. Israel et al. 1996). A prominent CO complex with no signs of massive star formation is located about $80 \mathrm{pc}$ south of N159.

In the two LMC areas observed, some $50 \mathrm{CO}(1-0)$ peaks have been identified (Johansson et al., in preparation). A smaller sample of these peaks and the one in the SMC (N27) have been investigated in higher transitions of $\mathrm{CO}$ and in the $\mathrm{CS}(2-1), \mathrm{HCN}(1-0)$ and the $\mathrm{HCO}^{+}(1-0)$ lines. The CO data have been analysed with a mean escape probability code assuming spherical clouds with uniform conditions ("MEP"-model in the following). The derived kinetic temperatures show a very good correlation with distance (on sky) to nearby $\mathrm{H}$ II regions, e.g. the highest temperature is found towards the very centre of the 30 Doradus nebula. Similarly, the higher the kinetic temperature the higher are the observed [C II]/CO and $\mathrm{HCO}^{+} / \mathrm{CO}$ emission ratios. The former ratios were taken from Israel et al. (1996) and are expected to be measures of the PDR-to-molecular-mass ratios. This ratio varies by a factor of about 200 from cloud to cloud while the range for the $\mathrm{HCO}^{+} / \mathrm{CO}$ ratios is less extreme, a factor of $\sim 15$. In contrast, the CS/CO and $\mathrm{HCN} / \mathrm{CO}$ ratios vary by no more than a factor of 3 , in line with expectations that those ratios reflect the conditions in denser and more shielded regions. It should be emphasized, however, that the samples indicating these trends are small (11 objects at most) at present. 


\section{Molecular abundance estimates}

\subsection{SOME GENERAL REMARKS}

Observationally, fractional abundances (abundances relative to that of hydrogen) are averages related to size-scales defined by the linear resolution of the observations or the line-of-sight extent of the gas. The resolution of the present observations is $10-15 \mathrm{pc}$ in the $3 \mathrm{~mm}$ range, similar to the extents of the largest $\mathrm{CO}$ complexes $(\sim 20 \mathrm{pc})$ in the Magellanic Clouds. This implies that the molecular emission observed is a conglomerate from regions of various chemical properties. More specifically, the fractional abundances presented here are averages over volumes defined by the $\mathrm{CO}(1-0)$ emission distribution on scales distinguishable by the beam. In accordance with Galactic work we call such volumes "clouds" but avoid the term "molecular clouds". This distinction is made to emphasize theoretical expectations that the $\mathrm{H}_{2}$ extents may significantly exceed those of $\mathrm{CO}$, particularly in the SMC, in contrast to the situation for Galactic clouds (see e.g. Maloney \& Black 1988). It is conceivable that similar differences exist on small scales ("clumps") as well as on larger scales ("clouds") or, in a scale-free medium, on all scales. Lequeux et al. (1994) suggest that the "interclump" hydrogen in the SMC is atomic rather than molecular, which further underlines the distinction above. Our definition of fractional abundances includes a possible contribution of atomic hydrogen within the $\mathrm{CO}(1-0)$ emitting volume, but any form of hydrogen outside the same volume is presumably missed. This is based on the assumption that the virial mass formula, with size and velocity dispersion defined by the $\mathrm{CO}(1-0)$ observations, provides a measure of the mass within the $\mathrm{CO}(1-0)$ boundary.

When comparing fractional abundances for different clouds, a major source of possible systematic errors is the estimate of total mass. Since we here focus on relative differences, the assumption that the virial mass is equal to the total mass can be relaxed to a linear relation. However, the degree of virial equilibrium may vary from cloud to cloud. Such a systematic effect may well be present, particularly in the violent region close to the 30 Doradus nebula. On the other hand, the very similar size-linewidth relations for the cloud samples in the 30 Doradus and the N159 areas (Johansson et al., in preparation) suggest a common equilibrium.

The determination of physical parameters, from which the molecular concentrations are derived, is also limited by the expected inhomogeneities of the gas. The uncertainties do not necessarily increase with increasing sizescales, but are determined by gradients present in the observed region. In this sense, derived parameters can actually suffer from larger errors on the smaller scales. It may be that molecular abundances derived in the Magellanic Clouds are more accurate, despite the lower resolution, than those 
derived for Galactic clouds. This would be true provided that the emissions are less saturated in the former case. The higher isotopic intensity ratios observed in the LMC than in Galactic and extragalactic sources suggest that this is the case (Johansson et al. 1994). In the limit of low optical depth the observed emission is, to a first approximation, proportional to the column density of the upper level and thus independent of the excitation temperature and its variations with location. On the other hand, determinations of total column densities require information on the excitation conditions. But again, average excitation properties should be more meaningful the smaller the optical depths are.

\subsection{DERIVED ABUNDANCES}

Table 1 lists four clouds which have been observed in a number of molecular transitions. The sample in the LMC covers a wide range of cloud properties as indicated by derived kinetic temperatures and observed [C II]/CO ratios: i) 30Dor, a cloud located close to the centre of the 30 Doradus nebula and the warmest cloud in the total sample of 11 objects, ii) N159W (associated with the H II region N159) is the cloud observed by Johansson et al. (1994) in the initial molecular line searches, and iii) the quiescent cloud N159S, located about $80 \mathrm{pc}$ south of N159. The fourth cloud is associated with the $\mathrm{H}$ II region $\mathrm{N} 27$ in the SMC.

TABLE 1. Cloud parameters

\begin{tabular}{|c|c|c|c|c|c|c|c|}
\hline \multirow[t]{2}{*}{ cloud } & \multirow{2}{*}{$\begin{array}{l}\mathrm{T}_{k i n} \\
{[\mathrm{~K}]}\end{array}$} & \multirow{2}{*}{$\begin{array}{l}\text { size } \\
{[\mathrm{pc}]}\end{array}$} & \multirow{2}{*}{$\begin{array}{l}\text { virial mass } \\
{\left[10^{4} \mathrm{M}_{\odot}\right]}\end{array}$} & \multicolumn{4}{|c|}{ luminosity ratios } \\
\hline & & & & {$[\mathrm{CII}] / \mathrm{CO}$} & $\mathrm{HCO}^{+} / \mathrm{CO}$ & $\mathrm{CS} / \mathrm{CO}$ & $\mathrm{HCN} / \mathrm{CO}$ \\
\hline 30Dor & 50 & 20 & 20 & 20 & 2.8 & 1.8 & 1.0 \\
\hline N159W & 20 & 17 & 14 & 1 & 1 & 1 & 1 \\
\hline N159S & 10 & 22 & 18 & 0.1 & 0.2 & 0.6 & 0.3 \\
\hline $\mathrm{N} 27$ & 15 & 20 & 12 & $\sim 1$ & 0.7 & 0.7 & 0.6 \\
\hline
\end{tabular}

Column densities have been derived using the MEP-code with fixed kinetic temperatures (column 2), defined by the CO data (see Section 2). The formal $2 \sigma$-errors for the kinetic temperature solutions are typically $20 \%$. For each cloud we have used the smallest $\mathrm{H}_{2}$ density required to obtain MEP-solutions for all observed transitions (about $10^{5} \mathrm{~cm}^{-3}$ in all cases). Cloud-sizes (half-power diameters) and virial masses are defined by the $\mathrm{CO}(1-0)$ data. The luminosity ratios listed in the last four columns are all normalized to those observed in the N159W cloud. 
Table 2 lists the relative differences (normalized to N159W) of fractional abundances, estimated in two ways: i) ratios of absolute fractional abundances $(\mathrm{X})$ using the virial mass as a measure of the hydrogen mass, and ii) ratios of column densities (N). The latter estimate is based on the assumption that clouds of similar sizes have similar masses.

With a possible exception of $\mathrm{HCO}^{+}$, the data listed in Table 2 show a striking uniformity of abundance ratios when each cloud is considered separately. This is probably a reflection of "composite chemistries" and is to be expected when abundances refer to size-scales as large as $20 \mathrm{pc}$.

TABLE 2. Underabundances relative to N159W

\begin{tabular}{|c|c|c|c|c|c|c|}
\hline \multirow[t]{2}{*}{ molecule } & \multicolumn{2}{|c|}{ N159S } & \multicolumn{2}{|c|}{ 30Dor } & \multicolumn{2}{|c|}{ N27 } \\
\hline & $\mathrm{X}$ & $\mathrm{N}$ & $\mathrm{X}$ & $\mathrm{N}$ & $\mathrm{X}$ & $\mathrm{N}$ \\
\hline $\mathrm{CO}$ & & & 3 & 2 & 4 & 5 \\
\hline CS & 1.5 & 1 & 5 & 3 & 4 & 4 \\
\hline SO & & & 9 & 6 & 5 & 7 \\
\hline $\mathrm{HCO}^{+}$ & 4 & 3 & 3 & 2 & 4 & 5 \\
\hline $\mathrm{HCN}$ & 2 & 1.5 & 7 & 5 & 5 & 5 \\
\hline HNC & 1.5 & 1.5 & 7 & 5 & & \\
\hline $\mathrm{H}_{2} \mathrm{CO}$ & & & 5 & 3 & 4 & 5 \\
\hline
\end{tabular}

The quiescent cloud, N159S, shows fractional abundances very similar to those in N159W. The formal differences are not significant.

The deficiencies derived for the SMC cloud have a very small scatter around the average, 4-5. Rubio et al. (poster, this conference) have observed three of the brightest CO-complexes in the SMC, including this one, in CS, $\mathrm{HCN}$ and $\mathrm{HCO}^{+}$lines. Their results are in close agreement with those presented here, indicating that the observed underabundances in the SMC are global. The physical properties of the N159W and N27 clouds seem to be similar, e.g. the [C II]/CO ratios point to the same fraction of PDR-volume (Table 1). This suggests that the general deficiencies are a reflection of the lower elemental abundances in the SMC. Relative to the LMC, the C, N and $\mathrm{O}$ abundances are down by factors between 2 and 6 in the SMC (Dufour 1984), strikingly similar to the derived molecular underabundances.

The 30Dor cloud, expected to be illuminated by an intense UV radiation field, shows underabundances of the same order as the SMC cloud(s). Although systematic errors may be more serious in this region (see Section 3.1), we do think that the observed deficiencies are real. This is supported by the fact that the $\mathrm{CO}$ emission is substantially weaker in the 30 Doradus area than in other areas in the LMC associated with prominent H II regions 
(see e.g. Israel et al. 1993). Provided the elemental abundances in the LMC are rather uniform on a global scale, the natural explanation for the deficiencies is higher molecular dissociation rates caused by the higher FUV fields in this area. However, such an explanation loses weight if the clouds in the outskirts of the nebula are considered. Those clouds are presumably less affected by the UV radiation, as suggested by the lower kinetic temperatures derived from $\mathrm{CO}$ data, smaller $\mathrm{HCO}^{+} / \mathrm{CO}$ ratios and their greater distances from the dominant $\mathrm{H}$ II region. The molecular line data collected towards these clouds are, at present, scarce but indicate underabundances similar to those observed in the central cloud.

To conclude, the molecular line data observed so far suggest that the fractional abundances within the 30 Doradus (central parts - outskirts) and the N159 (N159W and N159S) areas, separately, are rather uniform and independent of the UV illumination. This presumes that the effect of the $\mathrm{UV}$ field is revealed by the $[\mathrm{C} \mathrm{II}] / \mathrm{CO}$ and $\mathrm{HCO}^{+} / \mathrm{CO}$ ratios.

\section{3. $\mathrm{HCO}^{+}$}

As noted above, $\mathrm{HCO}^{+}$is the only molecule in our sample that may depart from the uniform fractional abundance differences observed from cloud to cloud. $\mathrm{HCO}^{+}$seems to be relatively more abundant than other molecules towards the 30Dor cloud (warm) and vice-versa in N159S (cold), when compared with $\mathrm{N} 159 \mathrm{~W}$ (intermediate kinetic temperature). In a larger sample, the $\mathrm{HCO}^{+} / \mathrm{CO}$ emission ratio shows the very same trend and, in a smaller sample, it follows the [C II]/CO ratio.

In dark cores, the formation of $\mathrm{HCO}^{+}$is dominated by a reaction of $\mathrm{H}_{3}{ }^{+}$ with $\mathrm{CO}$. However, there are alternative formation paths where $\mathrm{C}^{+}$plays an important role, see e.g. Sternberg \& Dalgarno (1995). Those authors present an elaborate model of the chemistry in dense PDRs. In a qualitative sense the observed $\mathrm{CS} / \mathrm{CO}$ and $\mathrm{HCO}^{+} / \mathrm{CO}$ emission ratios in the $\mathrm{LMC}$ are consistent with the model predictions: the CS abundance peaks in the core regions while $\mathrm{HCO}^{+}$, in addition to the core component, shows increased abundances in the outer layers where $\mathrm{C}^{+}$abundances are high. This is also consistent with the relatively large extent of the $\mathrm{HCO}^{+}$emission observed in N159W (Johansson et al. 1994). To decide whether this envelope component is responsible for the enhanced emission, in a relative sense, obviously needs further investigations.

\subsection{COMPARISONS WITH GALACTIC CLOUDS}

In comparisons of the chemical and physical properties of the interstellar medium in extragalactic sources, Galactic clouds are the natural reference objects. In general, however, Galactic molecular line surveys focus on par- 
ticular regions like e.g. high density cores. The relevant question in this context is how representative such regions are when global parameters are considered. An attempt to circumvent this problem was made by Johansson et al. (1994), where a remote Galactic molecular cloud associated with the $\mathrm{H}$ II region $\mathrm{S} 138$ was studied over its full $\mathrm{CO}(1-0)$ extent. Efforts were made to analyse the data in the same way as the LMC data and indicated a factor of 10 higher fractional abundances for the Galactic cloud relative those of N159W.

Extensive molecular line surveys have been done towards Orion and Sgr B2 (see Blake et al. (1987) and references therein) in essentially the same frequency ranges as observed in the Magellanic Clouds. In Orion we use the abundances for the extended ridge. This component is probably more representative in terms of dominant molecular line emission on global scales than the other more localized components. Sgr B2 was observed with a linear resolution of about $5 \mathrm{pc}$, thus approaching the resolution of the Magellanic Clouds observations ( $20 \mathrm{pc})$. However, it is well established that this beam covers at least three localized emission regions of quite different chemical composition (e.g. Hjalmarson \& Bergman 1992). On the other hand, this is to be expected for the Magellanic Clouds targets as well.

A straightforward comparison of the fractional abundances in $\mathrm{N} 159 \mathrm{~W}$ with those in the Orion ridge and Sgr B2, listed by Blake et al., gives results very similar to the above with respect to the general behaviour, although individual molecular abundances show a larger scatter. However, this agreement may be fortuitous since large systematic errors may be present in the estimates of hydrogen abundances. The data by Blake et al. rely on canonical values of the $\mathrm{CO} / \mathrm{H}_{2}$ abundance ratios, while observed $\mathrm{CO}-\mathrm{H}_{2}$ conversion factors were used by Johansson et al.. Thus more refined observations in combination with theoretical work are needed to determine the chemical differences in the Galaxy vs the Magellanic Clouds system.

\subsection{ISOTOPIC ABUNDANCE RATIOS}

In $\mathrm{N} 159 \mathrm{~W}$, the isotopomer emission ratios of $\mathrm{CS}, \mathrm{HCN}$ and $\mathrm{HCO}^{+}$are consistent with low optical depths in the observed transitions (Johansson et al. 1994). Heikkilä et al. (1996 and this conference) have observed the $\mathrm{HCO}^{+} / \mathrm{H}^{13} \mathrm{CO}^{+}$ratio in the SMC (N27), consistent with the results above, and detected $\mathrm{DCO}^{+}$and, tentatively, DCN in $\mathrm{N} 159 \mathrm{~W}$. The deuterated species have also been detected by Mauersberger et al. (this conference).

Heikkilä et al. derive an $\mathrm{HCO}^{+} / \mathrm{DCO}^{+}$abundance ratio of $60-180(2 \sigma-$ errors) and present estimates of kinetic temperatures and fractional electron abundances based upon this ratio. The kinetic temperature is constrained 
by the expected key reaction in the deuteration of $\mathrm{HCO}^{+}$,

$$
\mathrm{H}_{3}^{+}+\mathrm{HD} \rightleftharpoons \mathrm{H}_{2} \mathrm{D}^{+}+\mathrm{H}_{2}+\Delta E
$$

where the rate coefficient for the reverse direction is reduced by a factor $\sim$ $e^{-\Delta E / k T}$. The model results for the $\mathrm{HCO}^{+} / \mathrm{DCO}^{+}$ratio by Herbst (1982) lead to an upper limit to the kinetic temperature of $27 \mathrm{~K}$, consistent with the CO solutions. The estimate of the upper limit of fractional abundance of electrons, a few $\times 10^{-5}$, relies on a method introduced by Watson (1976), which balances the production and destruction (dissociative electron recombination) rates of $\mathrm{H}_{2} \mathrm{D}^{+}$. It should be emphasized that the latter estimate, in particular, suffers from large uncertainties in e.g. the electron recombination rate (cf. Adams \& Smith 1984, Larsson et al. 1996). The estimate refers to the most recent (higher) value of the recombination rate.

Another source of uncertainty is whether the $\mathrm{HCO}^{+}$and $\mathrm{DCO}^{+}$emissions sample the same volume: the formation of $\mathrm{DCO}^{+}$is favoured in cold regions while, as suggested above, some fraction of the $\mathrm{HCO}^{+}$emission may originate in the warmer zones of the PDRs. If this is the case, the estimated electron abundance is lowered in the $\mathrm{DCO}^{+}$emission region. We note, however, that this upper limit is approaching electron densities which may affect the excitation of molecules like $\mathrm{HCO}^{+}$and $\mathrm{HCN}$. This is of particular importance for the possible PDR envelope component of $\mathrm{HCO}^{+}$, arising in regions of significantly higher electron densities (see e.g. Sternberg \& Dalgarno 1995).

\section{Conclusions}

The molecular data presented here suggest similar underabundances in the SMC and in the 30 Doradus area, relative to the N159 clouds. In the SMC, this is probably a reflection of the lower elemental abundances, while the origin is more uncertain for 30 Doradus. It is questionable whether the higher UV field in 30 Doradus provide an explanation. Future observations should include a larger sample of clouds, in particular between 30 Doradus and N159 to investigate possible continuous changes of the abundances.

There are only a few chemical models published where attempts have been made to account for the distinct conditions of the ISM in the Magellanic Clouds. Millar \& Herbst (1990) allowed for the lower elemental abundances but the results apply to regions where the chemistry is not influenced by the FUV field. Models for CO (Maloney \& Black 1988, Lequeux et al. 1994) and the chemical model by Bel et al. (1986), in which the effects of the FUV field are considered, show better agreement with the observations.

It thus seems that PDR models are required to interpret the observational results. However, uniform plane-parallel models may be too simplistic when confronted with the low linear resolution data of the Magellanic 
Clouds, where the molecular emission is expected to arise from a large number of high density regions with a low volume filling factor (cf. Lequeux et al. 1994). Störzer et al. (1996) conclude that a spherical geometry becomes important when the PDR fills a large fraction of the cloud volume, a property that may characterise the ISM in the Magellanic Clouds to a larger extent than in the Galaxy. Spherical models with density gradients are probably needed to investigate chemical differences between the Galaxy and the Magellanic Clouds. Such models will also help to decide whether there is an extended component of the $\mathrm{HCO}^{+}$emission, of importance for the interpretation of the $\mathrm{HCO}^{+} / \mathrm{DCO}^{+}$ratios.

\section{References}

Adams, N.G., Smith, D. 1984, ApJ 284, L13

Bel, N., Viala, Y.P., Guidi, I. 1986, A\&A 160, 301

Blake, G.A., Sutton, E.C., Masson, C.R., Phillips, T.G. 1987, ApJ 315, 621

Booth, R.S. 1993, in New Aspects of Magellanic Cloud Research, eds. Baschek B., Klare G., Lequeux J., (Berlin: Springer) p.26

Dufour R.J. 1984, in Structure and Evolution of the Magellanic Clouds, IAU Symp. 108, eds. van den Bergh S., de Boer K.S., (Dordrecht: Reidel) p.353

Heikkilä, A., Johansson, L.E.B., Olofsson, H. 1996, A\&A, submitted

Herbst, E. 1982, A\&A 111, 76

Hjalmarson, §., Bergman, P. 1992, in Chemistry and Spectroscopy of Interstellar Molecules, eds. Bohme D.K., Herbst E., Kaifu N., Saito S., (University of Tokyo press) p.41

Israel, F.P., Johansson, L.E.B., Lequeux, J., Booth, R.S., Nyman, L.-Å., Crane, P., Rubio, M., de Graauw, Th., Kutner, M.L., Gredel, R., Boulanger, F., Garay, G., Westerlund, B. 1993, A\&A 276, 25

Israel, F.P., Maloney, P.R., Geis, N., Herrmann, F., Madden, S.C., Poglitsch, A., Stacey, G.J. 1996, ApJ 465, 738

Johansson, L.E.B., Olofsson, H., Hjalmarson, A., Gredel, R., Black, J.H. 1994, A\&A 291, 89

Koornneef, J. 1984, in Structure and Evolution of the Magellanic Clouds, IAU Symp. 108, eds. van den Bergh S., de Boer K.S., (Dordrecht: Reidel) p.333

Larsson, M., Lepp, S., Dalgarno, A., Strömholm, C., Sundström, G., Zengin, V., Danared, H., Källberg, A., af Ugglas, M., Datz, S. 1996, A\&A 309, L1

Lequeux, J., Le Bourlot, J., Pineau des Forêts, G., Roueff, E., Boulanger, P., Rubio, M. 1994, A\&A 292, 371

Maloney, P., Black, J.H. 1988, ApJ 325, 389

Millar, T.J., Herbst, E. 1990, MNRAS 242, 92

Mochizuki, K., Nakagawa, T., Doi, Y., Yui, Y.Y., Okuda, H., Shibai, H., Yui, M., Nishimura, T., Low, F.J. 1994, ApJ 430, L37

Poglitsch, A., Krabbe, A., Madden, S.C., Nikola, T., Geis, N., Johansson, L.E.B., Stacey, G.J., Sternberg, A. 1995, ApJ 454, 293

Rubio, M., Lequeux, J., Boulanger, F., Booth, R.S., Garay, G., de Graauw, Th., Israel, F.P., Johansson, L.E.B., Kutner, M.L., Nyman, L.-A. 1993, A\&A 271, 1

Sternberg, A., Dalgarno, A. 1995, ApJS 99, 565

Störzer, H., Stutzki, J., Sternberg, A. 1996, A\&A 310, 592

Watson, W.D. 1976, Rev. Mod. Phys. 48, 513 


\section{Discussion}

Rubio: Have you mapped these LMC clouds in other molecules than $\mathrm{CO}$ or have you used the derived size from the ${ }^{12} \mathrm{CO}$ map?

Johansson: In the $100 \mathrm{GHz}$ range, only $\mathrm{N} 159 \mathrm{~W}$ has been mapped in other molecules. In higher transitions, on the other hand, maps have generally been obtained yielding estimates of the emission extents. However, it should be emphasized that the derived fractional abundances are not very sensitive to the extents.

van Dishoeck: Could you comment on the [C I] $492 \mathrm{GHz}$ observations obtained with the $1 \mathrm{~m}$ AST/RO telescope at the South Pole?

Johansson: To my knowledge, three positions have been observed in the LMC and two detected. The detections are very weak, the peak intensities are at least a factor of 20 lower than the corresponding $\mathrm{CO}(1-0)$ intensities observed by SEST. There are very few observations of extragalactic [C I] but it seems that the [C I]/CO ratios in the LMC are low, in contrast to the [C II]/CO ratios. 